\title{
Caracterización fenotípica del bovino Criollo de Surinam en los distritos de Coronie y Nickerie
}

\author{
Tjon A San, G.G. ${ }^{1}$ y Molina-Flores, B. ${ }^{2}$
}

'Departamento de Producción y Sanidad Animal. Ministerio de Agricultura, Ganadería y Pesca. Surinam. ${ }^{2}$ División de Producción y Sanidad Animal. FAO. Roma. Italia.

\section{PalABRAS CLAVE ADICIONALES}

Surinam.

Bovino Criollo.

Caracterización fenotíica.

\section{RESUMEN}

El Criollo fue la primera raza bovina introducida en Surinam, pero las descripciones históricas apenas mencionan algunas de sus características fenotípicas. En el presente trabajo, fueron registradas las características fenotípicas de 105 bovinos Criollos de Surinam de diferentes grupos de edad. Las variables cualitativas descritas incluyen el patrón y color de la capa, tamaño y forma de los cuernos, posición y tamaño de la oreja, presencia y tamaño de morrillo o joroba y de la papada. Los valores cuantitativos incluyen el perímetro torácico, longitud escapulo-isquial, alzada a la cruz, longitud de la cara, longitud de la oreja, longitud del cuerno y el peso corporal. La mayoría de los datos obtenidos de los animales muestreados fueron bastante consistentes con las descripciones históricas, con la excepción de la enorme variación observada en el patrón y color de la capa, la desaparición total de la joroba y un ligero aumento del peso corporal.

\section{Phenotypic Characterization of the Surinamese Criollo cattle in the districts of} Coronie and Nickerie

\section{SUMMARY}

Although the Criollo was the first cattle breed introduced in Suriname, historical descriptions only mention a few of thephenotypic characteristics. In the present investigation, phenotypic characteristics of 105 Surinamese Criollo cattle of different age groups were recorded. The qualitative variables included color pattern, coat color, presence and shape of horns, ear shape and placement, presence of cervico-thoracic or thoracic hump, presence and size of dewlap. Quantitative values included heart girth, body length, height at withers, face length, ear length, length of the horns and calculated body weight. Most of the data obtained in the present investigation were fairly consistent with the historical descriptions, with the exception of the huge variation observed in coat pattern and color, the total absence of a hump and a slight increase in body weight.

\section{INFORMACIÓN}

Cronología del artículo.

Recibido/received: 03.04.15

Aceptado/Accept: 22.04.16

Online: 15.09 .16

Correspondencia a los autores/Contact e-mail:

g_tjonasan@hotmail.com

\section{INTRODUCCIÓN}

Los primeros bovinos llegados al Nuevo Mundo vinieron con los exploradores españoles provenientes de la Península Ibérica, multiplicándose en un principio en la isla de La Española y extendiéndose después al resto del continente, estableciéndose también en Surinam (Bastiaensen et al., 1995). En los siglos que siguieron, el bovino Criollo de Surinam, se vio influenciado primero por la introducción de razas británicas y más tarde por razas holandesas y, en menor medida por las cebuínas. No fue hasta finales del 1950 cuando comenzaron a implementarse los primeros programas de mejoramiento mediante el uso de razas exóticas. Esto dio lugar a la pérdida del fondo genético del bovino Criollo de Surinam, cuyos efectivos se vieron reducidos a tan solo 3341 animales, representando menos del $12 \%$ de la cabaña bovina nacional, según el V Censo Agrícola Nacional realizado entre 2008 y 2009 (Anonymous, 2014).

Debido a que los programas de mejoramiento se implementaron principalmente en las zonas rurales más cercanas a Paramaribo, capital de la nación, el impacto de estas razas exóticas en los rebaños de Criollo de 
Surinam de los distritos de Coronie y Nickerie, al oeste del país, fue mínimo. Estos distritos se dedican fundamentalmente a la producción de arroz, por lo que tradicionalmente el ganado bovino se empleaba en los trabajos agrícolas, pero también proveía a la familia de leche fresca y, esporádicamente, de carne. Además la raza tenía un papel socio-económico importante, pues servía como cuenta de ahorro y era práctica común intercambiar animales en concepto de dote durante los matrimonios. Los ganaderos todavía practican el pastoreo comunal, y sólo las vacas en fase final de gestación, terneros y animales destinados a la venta, son llevados a las parcelas agrícolas para pastar en los rastrojos de campos de arroz y limpiar de malas hierbas los canales de riego, recibiendo también algún suplemento alimenticio compensatorio.

El bovino Criollo de Surinam está muy bien adaptado a las condiciones típicas del trópico húmedo y de los sistemas agrícolas de bajos insumos practicados por la mayoría de los agricultores del país. Localmente la raza es reconocida por poseer atributos valiosos para la producción de carne y leche, ya sea como raza pura o a partir de sus cruces. Sin embargo, poco se ha escrito sobre su apariencia y sus características productivas. Según la Enciclopedia de Surinam, el ganado bovino Criollo del país es pequeño, con un peso adulto de unos $250 \mathrm{~kg}$, presenta capas que van del amarillo al rojo y posee orejas pequeñas y cuernos de tamaño medio. También pueden presentar una pequeña joroba cervical y una papada poco desarrollada (Bruijning et al., 1977). El presente trabajo pretende estudiar las características fenotípicas del bovino Criollo de Surinam y compararlas con su descripción histórica, como parte de un trabajo más extenso que tiene por meta la caracterización, conservación y revalorización de la raza.

\section{MATERIAL Y MÉTODOS}

Utilizando los datos del V Censo Agrícola Nacional, se identificaron las explotaciones con ganado bovino Criollo de Surinam a muestrear en los distritos de Coronie y Nickerie. Con el fin de confirmar la presencia de ani- males de dicha raza en estas explotaciones, se organizaron varias visitas de campo en colaboración con los oficiales de salud animal y de extensión ganadera de estos distritos, los cuales seleccionaron, de acuerdo con sus criterios personales, un total de 105 animales ( 4 machos y 101 hembras) de 2 años de edad como mínimo. La recolección de los datos se realizó directamente en el campo, una vez los animales fueron inmovilizados por sus propietarios. Las características fenotípicas a evaluar se dividieron en variables cualitativas y cuantitativas, de acuerdo con las directrices de la FAO (2012). Las variables cualitativas se obtuvieron mediante la observación directa del animal. Para el registro de las variables cuantitativas se utilizaron un bastón zoométrico y una cinta métrica calibrados en centímetros. Para calcular el peso corporal de los animales se utilizo la fórmula de Shaeffer (Sastry et al., 1982).

\section{RESULTADOS Y DISCUSIÓN}

Se encontró una gran diversidad en el color de la capa del bovino Criollo de Surinam actual. El patrón de capa predominante fue el uniforme o de un solo color $(69,52 \%)$, seguido del manchado, atigrado, pintado, cabeza-blanca y cinturón-blanco (18,10 \%, 4,76 \%, 3,81 \%, $2,86 \%, 0,95 \%$ ). Se han observado al menos 18 colores diferentes en las capas de los animales, de los cuales el 34,28 \% tenía una capa uniforme de color marrón, variando del marrón claro al marrón muy oscuro y, por lo general, con una franja de color marrón más claro en la línea dorso-lumbar; el 18,10 \% tenía un pelaje compuesto de color marrón y blanco; el 14,29 \% tenía una capa uniforme de color beige (amarillo); el 14,29 \% presentaba un color de la capa predominantemente rojo, variando del rojo claro al rojo muy oscuro, y rojo y blanco. Otros colores de la capa eran el negro uniforme $(7,62 \%)$, varios tonos de gris (5,71\%), negro y blanco (3,81\%) y blanco (1,90\%). Sólo dos de los animales muestreados (1,90\%) carecían de cuernos de forma natural, todos los demás animales tenían cuernos. La forma predominante de los cuernos era en gancho $(56,19 \%)$, con los pitones apuntando hacia arriba y hacia delante mayoritariamente, seguido de cuernos en forma de lira

Tabla I. Media y desviación estándar de los caracteres cuantitativos (Mean and standard deviation of the quantitative characteristics).

\begin{tabular}{|c|c|c|c|c|c|}
\hline \multirow[b]{2}{*}{ Parámetro } & \multicolumn{2}{|c|}{ Grupo X } & \multirow{2}{*}{$\begin{array}{c}\text { Grupo Y } \\
\text { Solo hembras }\end{array}$} & \multirow{2}{*}{$\begin{array}{c}\text { Grupo Z } \\
\text { Solo hembras }\end{array}$} & \multirow[b]{2}{*}{ Muestra total } \\
\hline & Machos & Hembras & & & \\
\hline Perímetro torácico & $142,63 \pm 31,30$ & $152,76 \pm 12,32$ & $160,21 \pm 9,23$ & $160,29 \pm 9,24$ & $157,64 \pm 12,03$ \\
\hline Longitud escáp.-isq. & $133,25 \pm 14,66$ & $127,67 \pm 12,88$ & $136,85 \pm 9,31$ & $135,67 \pm 11,68$ & $134,06 \pm 11,60$ \\
\hline Alzada a la cruz & $112,70 \pm 6,36$ & $115,63 \pm 6,82$ & $118,18 \pm 6,02$ & $117,56 \pm 6,52$ & $117,10 \pm 6,47$ \\
\hline Longitud cara & $49,00 \pm 1,41$ & $51,13 \pm 3,63$ & $52,28 \pm 3,32$ & $52,81 \pm 4,14$ & $51,99 \pm 3,63$ \\
\hline Longitud oreja & $21,50 \pm 0,58$ & $21,89 \pm 2,76$ & $22,40 \pm 2,22$ & $21,94 \pm 2,32$ & $22,12 \pm 2,35$ \\
\hline Longitud cuerno & $18,25 \pm 6,98$ & $20,50 \pm 6,90$ & $29,38 \pm 6,78$ & $33,34 \pm 9,50$ & $27,58 \pm 9,05$ \\
\hline Peso corporal & $251,79 \pm 89,71$ & $277,48 \pm 69,58$ & $323,45 \pm 50,44$ & $321,23 \pm 54,22$ & $308,35 \pm 61,78$ \\
\hline
\end{tabular}


$(27,62 \%)$, rizados $(8,58 \%)$, rectos $(3,81 \%)$ e incompletos o tocones $(1,90 \%)$. Todos los animales muestreados presentaban orejas con una disposición horizontal en el 99,04 \% de los casos, mientras que tan solo un animal mostraba orejas caídas (0,95\%). Ninguno de los animales muestreados presentaba morrillo o giba. Las hembras de más edad sí mostraban un engrosamiento en la zona del cuello, pero no se podía clasificar como una joroba cervical. Más de la mitad de los animales incluidos en la muestra tenía una pequeña papada (60\%) y estaba ausente en el resto de los animales.

Los animales muestreados fueron divididos en tres grupos atendiendo a la edad de estos: animales de 2 años a menos de 5 años (grupo X), de 5 años a menos de 9 años (grupo Y) y animales mayores de 9 años (grupo Z). Se calcularon la media y la desviación estándar, para los diferentes grupos de edad, de las medidas observadas para el perímetro torácico, la longitud escapuloisquial (distancia del segmento recto comprendido entre el encuentro y la punta de la nalga), alzada a la cruz, longitud de la cara, longitud de la oreja y longitud del cuerno (en centímetros) y del peso corporal estimado (en kilogramos), los cuales se muestran en la tabla I.

\section{CONCLUSIONES}

El bovino Criollo de Surinam actual difiere en cierta medida del descrito históricamente. Se encontró una mayor variabilidad en el patrón y el color de la capa. La presencia y el tamaño de la papada coinciden con la descripción histórica, pero no la presencia de joroba, que no estaba presente en ningún animal. Aunque, el promedio de la longitud escápulo-isquial $(134,06 \pm 11,60)$ y de la alzada a la cruz $(117,10 \pm 6,47)$ efectivamente indican un animal de pequeñas dimensiones, el promedio de peso corporal $(308,35 \pm 61,78)$ es ligeramente superior al de las descripciones anteriores. La longitud media de la oreja $(22,12 \pm 2,35)$ y de la cara $(51,99 \pm$ $3,63)$, indican orejas relativamente pequeñas, coincidiendo con la descripción histórica. La longitud media del cuerno $(27,58 \pm 9,05)$ indica cuernos de tamaño medio, lo que también coincide con la descripción histórica. Posteriores estudios morfo-estructurales y fanerópticos más exhaustivos deberían desarrollarse para, sobre la base de los resultados obtenidos, definir estándares raciales, los cuales convendría apoyar con estudios de caracterización molecular y filogenéticos que permitirán, en primer lugar, verificar la identidad racial del Criollo de Surinam y, en segundo lugar, proporcionar una mayor comprensión de las relaciones genéticas con otras razas americanas y europeas.

\section{AGRADECIMIENTOS}

Un especial agradecimiento por su asistencia en el campo a Lucia Dulder, Ronny Kranenbrug, Rudy Asmoredjo y Steve Brug.

\section{BIBLIOGRAFÍA}

Anonymous. 2014. Eindrapport van de vijfdelandbouwtelling 2008-2009. Ministerie van Landbouw, Veeteelt en Visserij. Paramaribo. Suriname.

Bastiaensen, P.; de la Marche, L.; Koenraadt, G. und Van de Gejuchte, E. 1995. Veeteeltgids voor Suriname - Herkauwers. Leo Victor. Paramaribo. Suriname.

Bruijning, C.F.A.; Voorhoeve, J. and Gordijn, W. 1977. Encyclopedie van Suriname. Elsevier. Amsterdam. Nederland. pp. 372-373; 624-625.

FAO. 2012. Phenotypic characterization of animal genetic resources. FAO Animal Production and Health Guidelines. $N^{\circ}$ 11. Rome. pp. 87-94.

Sastry, N.S.R.; Thomas, C.K. and Singh, R.A. 1982. Farm animal management and poultry production. Vikas Publishing House. New Delhi. India. 